\title{
la lengua guaymí en Costa Rica
}

José Manuel Murillo Miranda ${ }^{1}$

Universidad Nacional, Costa Rica

\section{RESUMEN}

Como aproximación panorámica al estudio actual del guaymí, una de las lenguas de la familia chibcha, es un análisis introductorio de los rasgos gramaticales y tipológicos generales de esa lengua. Previa información de índole antropológica e histórico-cultural, se exponen en forma analítica aspectos sintácticos, sobre la frase verbal, el morfema de negación, el de reflexivización, los sujetos dativos, los objetos directos. Señala algunas tareas pendientes, que suponen un estudio más pormenorizado y extendido.

\section{Abstract}

Providing a panoramic view of the present studies of the Guaymí language, one of the Chibchan languages, this is an introductory analysis of the general grammatical and typological features of that language. First, information is given on anthropological, historical and cultural aspects. Then syntactic features are described for the verb phrase, the morpheme for negation, reflexive forms, dative subjects and direct objects. Mention is made of pending tasks requiring a broader and more detailed study.

Palabras clave: guaymí, chibcha, lenguas indígenas, sociolingüística. Key words: Guaymí, Chibcha, indigenous languages, sociolinguistics.

l Correo electrónico: jomuri@ racsa.co.cr 


\section{Introducción ${ }^{2}$}

\section{Determinación geográfica y genética}

El guaymí es una lengua de la familia chibcha, hermanada con el bocotá dentro del grupo guaymí, incluido en la subrama ístmica de larama sureña ${ }^{3}$. Es originaria de Panamá, donde se encuentra el mayor número de hablantes, estimados en más de 100.000 individuos, ubicados en la comarca Ngäbe-Buglé, cuya población, para el 2004, estaba estimada en 128.978 personas ${ }^{4}$. Esta comarca fue fundada en 1997 en las provincias panameñas de Bocas del Toro, Chiriquí y Veraguas; posee un estatus jurídico especial; las leyes y constitución panameñas no tienen jurisdicción en esa zona; y se rige por una junta directiva que promulga y ejecuta leyes autóctonas.

\section{Datos generales de los guaymíes en Costa Rica}

Los indígenas guaymíes (autodenominados ngäbe) empezaron a asentarse en el territorio costarricense al tenor de una serie de migraciones que comenzaron en la década de 1950 desde Panamá. La oleada inicial consistió de unas cinco familias que se establecieron entreel río Limoncito y la divisoria de aguas de la Fila Cruces, en Coto Brus, Puntarenas. En 1977, ocurre una nueva migración desde Bocas del Toro, Veraguas y Chiriquí. Aparte de la población ya estabilizada, también se presenta una población flotante, la cual permanece meses e incluso años ${ }^{5}$. Los guaymíes, dado lo anterior, siempre se consideraron

2 Una versión preliminar de esta primera parte fue presentada en el I Congreso Intemacional de Lingüística Aplicada, llevado a cabo en octubre de 2007, en el Campus Omar Dengo, de la Universidad Nacional de Costa Rica.

3 Adolfo Constenla Umaña, Las lenguas del área intermedia: introducción a su estudio areal (San José: Editorial de la Universidad de Costa Rica, 1991).

4 Salvatierra Salazar, El indígena costarricense: una visión etnográfica (1 ${ }^{\mathrm{a}}$. ed., 2002) (Cartago: Editorial Tecnológica de Costa Rica, 2006).

5 José Rafael Acuña Vargas y Freddy Calvo Rodríguez, Aspectos geográficos y socioculturales de la Reserva Indígena Guaymí de Coto Brus, Puntarenas. Tesis de licenciatura. (Heredia: Universidad Nacional, 2002). 
extranjeros. Arroyo no menciona el guaymí como lengua indígena nacional; incluye el bribri, el cabécar, el térraba, el boruca y el guatuso $^{6}$. No fue sino hasta 1991, con la Ley de Inscripción y Cedulación Indígena (Ley 7225), cuando se declaró que los guaymíes asentados en territorio nacional son ciudadanos costarricenses?

En Costa Rica, la población guaymí está ubicada en la provincia de Puntarenas, en las siguientes cinco reservas:

1. Reserva Indígena Abrojos Montezuma: Provincia de Puntarenas, Cantón de Corredores, Distrito Corredor.

2. Reserva Indígena Conteburica: Provincia de Puntarenas, ente los Cantones de Corredores y Golfito, Distritos Laurel y Pavón.

3. Reserva Indígena de Coto Brus: Provincia de Puntarenas, Cantones de Coto Brus y Buenos Aires, Distritos Limoncito y Chánguena.

4. Reserva Indígena Guaymí de Alto Laguna de Osa: Provincia de Puntarenas, Cantón de Osa, Distrito Sierpe.

5. Reserva Indígena de Altos de San Antonio: Provincia de Puntarenas, Cantón de Corredores, Distrito Canoas. Declarado territorio indígena el 16 de mayo del 2001 mediante el decreto 29451$\mathrm{G}$, publicado en la Gaceta, $n^{\circ} 93^{8}$.

Según el censo de $2000^{9}$, la población guaymí residente en las reservas es del $3.9 \%$ de la población indígena nacional, para un total de 2.563 personas $^{10}$, mientras que el total nacional es de 5.370 individuos, según Acuña y Calvo ${ }^{11}$.

6 Víctor Manuel Arroyo, Lenguas indígenas costarricenses, 2." edición (San José: EDUCA, 1972).

7 Joel Mora Maroto y Oscar Almengor Fernández, "Proyecto de un nuevo Estatuto Indígena para Costa Rica: experiencias y desafíos", <http://www.oit.or.cr/unfip/estudios/estaindcr.htm>, 2002.

8 Acuña y Calvo.

9 Deisy Corrales Díaz, "Desarrollo humano en salud en comunidades indígenas", <www.ministeriodesalud.go.cr/ops/ documentos>, 2005.

10 Elizabeth Solano Salazar, "La población indígena en Costa Rica según el censo 2000", <www.ccp.ucr.ac.cr/noticias/ simposio/pdf/solano.pdf>, 2004.

11 Acuña y Calvo, 76. 
La población guaymí es básicamente agrícola, y su producción es sólo de subsistencia. Por lo general, son los no indígenas quienes logran acumular pequeños excedentes. Los productos más cultivados son la yuca, el coco, arroz, frijol, ñame, plátano, ñampí, pejibaye, cacao, banano y tiquisque. Su principal fuente de carne consiste en gallinas, patos, pavos y cerdos, además de lo provisto por la práctica de la cacería y de la pesca, como conejos, iguanas, ardillas, sábalos, mojarras y camarones de río. Sin embargo, los pequeños propietarios a veces no pueden asegurar una provisión constante y variada de sus parcelas, por lo que deben emplearse como jornaleros de no indígenas, sea en labores agrícolas o recolecta de café (con el consiguiente desplazamiento fuera de las reservas) para obtener dinero y satisfacer las necesidades primarias.

Tradicionalmente, la vivienda guaymí era circular, construida de palma, con piso de tierra y techo cónico; las viviendas familiares suelen estar separadas entre sí por mucha distancia. No obstante, por influencia criolla, ahora se usa madera de aserradero para las paredes y láminas de zinc en el techo. El mobiliario consiste en hamacas, bancos toscos y un "jarrón" o "ático". En los núcleos poblacionales grandes, como en Limoncito, las casas se hallan más cerca unas de otras; son prefabricadas o de madera, con piso rojo, y con servicios de agua y electricidad, electrodomésticos y, para los más afortunados, reproductores de DVD y computadora ${ }^{12}$.

En cuanto a su organización social, los guaymíes exhiben un tipo de familia que se rige por el matrimonio poligínico; es decir, que un hombre rico y próspero, para ser considerado como tal, puede tener varias esposas. Acuña y Calvo indican que los individuos con más de una esposa, como los caciques, toman la precaución de mantenerlas separadas en sendos ranchos para evitar conflictos ${ }^{13}$. El tipo de matrimonio es el de intercambio; los potenciales consuegros acuerdan

12 Presidencia de la República, Consejo Social, "Grupos étnicos existentes en Costa Rica", <http:// www.ifam.go.cr/PaginaIFAM/docs/pobindigena.pdf>, 2004.

13 Acuña y Calvo, 88-89. 
dar un hijo o hija en edad casadera (catorce o quince años en promedio) al otro cuando sea la ocasión. El matrimonio poligínico se encuentra en franco desuso entre las nuevas generaciones, por influencia de la cultura mestiza costarricense y por la legislación sobre pensiones alimenticias, la cual inhibe a muchos hombres a tener hijos con más de una mujer.

La vestimenta femenina, en la mayoría de los casos, consiste de largos batones muy coloridos, fácilmente reconocibles. Originalmente, el material usado era nogwata ('mastate'), pero poco a poco fue cambiando por tela ${ }^{14}$. La vestimenta masculina está muy occidentalizada y no se diferencia en nada de la indumentaria de los blancos; sin embargo, un elemento de exclusivo uso masculino es un tipo de collar llamado "chaquira", elaborado con huesos, cuentas de vidrio y conchas, siguiendo dif erentes patrones. Dadas las condiciones del terreno y la precaria condición de los caminos, es común que las personas del lugar usen botas de hule y, por lo general, la costumbre es dejar las botas a la entrada de la casa y caminar descalzo en ella para no ensuciarla.

\section{El estado de la lengua guaymí en Costa Rica}

Según Quesada Pacheco (ver artículo en este número), en Panamá los guaymíes, por ser el grupo indígena más numeroso, tienen una gran vitalidad cultural y lingüística. Poseen una estación de radio (básicamente religiosa), los jóvenes hablan fluidamente su lengua. Además existe una importante producción de textos que recopilan historias tradicionales y otros enfocados a la enseñanza-aprendizaje del guaymí, con un trasfondo religioso cristiano. Asimismo se realizan esfuerzos de instituciones gubernamentales por conservar y promover su cultura tradicional. Incluso en este momento se están realizando esfuerzos para crear una universidad étnica en la comarca

14 Instituto de Estudios de las Tradiciones Sagradas de Abia Yala (IETSAY), Narraciones ngäbes: revitalización de la cultura tradicional (San José: Fundación Coordinadora de Pastoral Aborigen, 1997). 
Ngäbe-Buglé; este investigador está en contacto con uno de los líderes de esta empresa, con quienes se quiere realizar proyectos conjuntos.

En Costa Rica la lengua guaymí muestra indicios de vitalidad. Bauman tiene una conocida clasificación para analizar el estado en que se encuentran las lenguas indígenas con respecto al inglés en Estados Unidos: 1.florecientes, 2. duraderas, 3. declinantes, 4. obsolescentes, y 5. extintas $^{15}$. Seguidamente se enumeran los parámetros utilizados por Bauman para categorizar las lenguas, así como una valoración del desempeño de los hablantes de la Reserva Guaymí en términos de esos parámetros; en paréntesis aparecen los valores obtenidos mediante observación directa:

- $\quad$ Edad de los hablantes y su grado de bilingüismo (+): los niños exhiben bilingüismo para sus mayores y sus iguales.

- Proporción de hablantes con respecto al total de la población (+): si bien existe bilingüismo, en muchos casos en detrimento del guaymí, todos los hablantes entrevistados conocen la lengua.

- $\quad$ Fluidez de los hablantes jóvenes ( \pm ): entre menos contacto con el español, mayor fluidez, y ello depende más de la cercanía a los centros urbanos que a la edad.

- $\quad$ Grado de preferencia por la lengua materna ( \pm ): la lengua es hablada tanto por ancianos como por niños, aunque si la conversación gira en torno a temas no propios de su entorno o cultura, se usa el español. No obstante, en presencia de un sulia ('blanco') la lengua no tiende a ocultarse.

- $\quad$ Grado de bilingüismo ( \pm ): todos son bilingües; en la Reserva Guaymí no se encontraron hablantes monolingües.

- Capacidad de adaptación de la lengua a una cultura cambiante (+): si bien hay muchos hispanismos, los hablantes se enfrentan al mundo con su lengua; de hecho, recientemente un maestro de

15 James J. Bauman, A Guide to Issues in Indian Language Retention (Washington: Center for Applied Linguistics, 1980). 
primaria elaboró un texto para la enseñanza de las matemáticas en esa lengua y un libro sobre biodiversidad.

En general, cuanto más alejados se encuentran de los núcleos poblacionales principales, su uso del español es más escaso y el de guaymí más intenso. De ello se desprende que la lengua guaymí en Costa Rica, por el momento, no está gravemente amenazada y su grado de vitalidad en la escala de Bauman bien puede calificarse de duradero.

\section{Estudios previos}

Los trabajos de descripción gramatical del guaymí son escasos. El tipo de producción bibliográfica que ha tenido más desarrollo desde hace tres lustros ha sido el de alfabetos ilustrados ${ }^{16}$, textos de prelectura, lectura y textos pedagógicos de guaymí como lengua materna para educación primaria ${ }^{17}$. Tales escritos son elaborados principalmente por organizaciones indígenas, con la ayuda de agencias gubernamentales, universidades estatales, grupos religiosos y organizaciones internacionales como UNESCO ${ }^{18}$. Mucha de esta producción procede de Panamá, pero también hay publicaciones en Costa Rica. Dentro de ese tipo de producción textual también se pueden incluir diccionarios bilingües ngäbe-español ${ }^{19}$ y obras de cultura e historias tradicionales ${ }^{20}$.

La tarea de recolección de vocabulario y confección de diccionarios por parte de estudiosos no guaymíes incluye los trabajos de

16 Adolfo Constenla Umaña y Leonicio Bejarano, Alfabeto práctico ilustrado guaimí (San José: Despacho del Ministro de Educación Pública, 1994).

17 Adolfo Constenla Umaña, Tärä ngäbere: Libro guáimi (San José: Comité de Educación de la Comisión Costarricense de Cooperación con la UNESCO, 2004).

18 Carmen Rojas Chaves, "La enseñanza de las lenguas indígenas en Costa Rica". Departamento de Educación Indígena, Ministerio de Educación Pública, <www.unesco.or.cr/portalcultural/ lenguasl.pdf $>, 2007$.

19 Luciano Sonso Javilla, Diccionario ngäbe-español (Panamá: Oficina de la UNESCO para Centroamérica y Panamá, 1997). 
Franco y de Alphonse, los cuales son descripciones gramaticales hechas sobre la base de paradigmas de la época ${ }^{21}$; son de poca utilidad para una descripción gramatical moderna por cuanto consisten en mera recopilación de vocabulario, el primero, y en una transferencia de categorías de lenguas indoeuropeas al guaymí, el segundo. No fue sino hasta el último cuarto del siglo xx cuando aparecieron descripciones más modernas, las cuales, sin embargo, no exhiben la profundidad requerida de una gramática referencial. Entre estas se encuentran las de Kopesc y Kopesc, Lininger, Arosemena y de $\mathrm{Abarca}^{22}$, todas las cuales corresponden a estudios fonológicos (principalmente inventarios). El primero establece ocho fonemas vocálicos, dieciocho consonánticos y dos suprasegmentales (acento y nasalización); el segundo propone un fonema de labialización/w/opuesto a un fonema semiconsonántico /w/; Abarca argumenta que el fonema semiconsonántico no es tal, sino alóf ono de la/u/, además propone que la lengua manifiesta tono alto y tono bajo. Por su parte, Arosemena y Arosemena ${ }^{23}$, realizan un estudio sobre aspectos discursivos de la lengua en mención, como interacción de participantes, especialmente enfocado a la narración.

20 IETSAY.

21 Padre Blas José Franco, "Noticias de los indios del depto. de Veragua, y vocabularios de las lenguas guaymí, norteño, sabanero y dorasque”. Alphonse Louis Pinart, (Ed.), Colección de lingiuística y" etnografía americanas (San Francisco, CA: A. L. Bancroft, 1882). E. S. Alphonse. Guaymí Grammar and Dictionary with Some Ethnological Notes (Washington D.C.: Bureau of American Ethnology, 1956).

22 Bonnie M. Kopesec y Michael F. Kopesec, "La jerarquía fonológica del guaymí.” Patricia Baptista (Red.), Lenguas de Panamá. Tomo I. Sistemas fonológicos. Instituto Lingüístico de Verano e Instituto Nacional de Cultura. Panamá: Imprenta de la Nación, 1974) 17-30. Barbara Lininger Ross, "Estudios sobre el Guaymí Ngabere: Fonología, alfabeto y diccionario provisional". Revista de Filología y Lingüística de la Universidad de Costa Rica VII, 1-2 (1981). Melquíades A. Arosemena B. "Principales rasgos fonológicos del guaymî'. Revista Latinoamericana de Estudios Etnolingüísticos 3 (1983): 87-119; este documento y otro de Michael Kopesec (1975) "Los elementos verbales y sustantivos y la oración guaymî" no estaban disponibles para el autor al elaborar este artículo. Rocío Abarca González, “Análisis fonológico de guaymí movere”. Estudios de Lingüística Chibcha. San José: Programa de investigación del Departamento de Linguística de la Universidad de Costa Rica, tomo IV (1985): 7-46.

23 Melquíades Arosemena B. y Frances C. Arosemena, Estudios sobre el discurso en guaymí. Lènguas de Panamá, 8. (Panamá: Instituto Lingüístico de Verano, 1980). 


\section{Tanto Constenla como Margery recogen y estudian narraciones} tradicionales, en una línea de trabajo más etnolingüística ${ }^{24}$.

Payne hace una descripción de las formas en que los sujetos son marcados. Para el autor, los sujetos se pueden marcar con los siguientes elementos: gwe, e, biti y bötö. Payne concluye que la noción de sujeto en guaymí, un caso de ergativo extendido, se rige bajo el principio de "participante central", entendido como el protagonista de la situación planteada en la oración ${ }^{25}$. Payne indica que el guaymí presenta una marcación de ergativo extendido en los pretéritos, por un lado, aunada a una escisión de tipo TAM, donde la marca de ergatividad se limita al tiempo pasado ${ }^{26}$. Estas mismas condiciones son expuestas por Quesada ${ }^{27}$.

\section{Propiedades gramaticales del guaymí}

\section{Características tipológicas ${ }^{28}$}

La lengua guaymí es una lengua cuyo orden básico es SOV, para las oraciones transitivas (1) y SV para las oraciones intransitivas (2). Otros tipos de complementos en la oración, como los que codifican situaciones locativas y temporales, son posverbales, como la frase

24 Adolfo Constenla Umaña. "Seis narraciones tradicionales guaimíes (moves)", Revista de filología y lingüística de la Universidad de Costa Rica VIII, 1-2 (1982): 103-107; y Enrique Margery Peña, "La narrativa oral de guaymíes y bocotás como expresión de un 'área de habla' en el extremo occidental de Panamá". Estudios de Lingiiística Chibcha. San José: Programa de investigación del Departamento de Linguística de la Universidad de Costa Rica. Serie anual. XVI-XVII (1997-98).

25 Thomas Payne, "Subject in Guaymi. Estudios varios sobre las lengua chibchas de Costa Rica". San José: Programa de Investigación del Departamento de Linguiística de la Universidad de Costa Rica, I (1982): 69.

26 Thomas Payne, Describing Morphosyntax: A Guide for Field Linguistics (Cambridge: Cambridge University Press, 1997) 160-161.

27 Juan Diego Quesada, The Chibchan Languages (Cartago: Editorial Tecnológica de Costa Rica, 2007).

28 En esta descripción se utilizan categorías de Givón y de Greenberg. y se tiene en cuenta el estudio de Smith y Zamora. Talmy Givón, Syntax. Vol. 2 (Philadelphia, PA: John Benjamins, 2001); Joseph Greenberg, Universals of Language (Boston, MA: MIT Press, 1986); Heidi Smith y Teresita Zamora, "Un análisis tagmémico del dialecto nobere del guaymî", en Revista de Filología y Lingüística de la Universidad de Costa Rica V, 1-2 (1979): 125-137. 
posposicional en (3). No obstante, es posible encontrar sujetos colocados de manera posverbal, como en (4). De todas maneras, tal como ocurre con otras lenguas emparentadas, el orden estructural más importante es OV.

(1) krua gwe chibo kämi-ri tigre ERG cabra matar-IRREMOTOSPECTIVO 'El tigre mató a la cabra'

(2) kwe gwe jadug-abare $b o-b u$

3SG ERG dormir-REMOTOSPECTIVO CFL.DÍA-dos 'Él durmió por dos días'

(3) kue tä ümá den ñö te

3SG EXIST arena agarrar.PRS río en 'Él está agarrando arena en el río'

(4) monso met-ani kwe gwe Niño golpear-IRREMOTOSPECTIVO 3sG ERG 'Él golpeó al niño'

\section{La frase nominal}

La frase nominal guaymí se compone de un núcleo nominal obligatorio, que puede ser un sustantivo simple, como en el sujeto transitivo y en el objeto directo de (5), un demostrativo, tal como se ve en el sujeto de (6) o un pronombre personal, más un conjunto de modificadores posnominales, como los adjetivos calificativos (7), los numerales (8) y los demostrativos (9). El orden básico de los componentes de la frase nominal mencionados es sustantivo-adjetivo-demostrativo, y cuando aparece un numeral, éste antecede al adjetivo, como se muestra en (10). 
(5) kälu kriägä bätete

Lluvia hojas lavar.PRS

'La lluvia lava las hojas'

(6) Se nu tú

DEM perro diente

'Esos son dientes de perro'

(7) mugue kabre

estrella mucha

'muchas estrellas'

(8) ju kwä-rige mä käi te

casa CFL.REDONDO-cinco 2SG pueblo en

'Hay cinco ranchos en tu pueblo'

(9) dän kira ne bätete

ropa vieja DEM lave.PRS

‘Lave esta ropa vieja!’

(10) kwe gwe kwí ku-bú krigri ri-ri

3SG ERG gallina CFL.REDONDO-dos grande cocinar-IRREMOTOSPECTIVO

'Ella cocinó dos gallinas grandes'

La relación de posesión se realiza anteponiendo el elemento nominal (que puede ser un sustantivo pleno (11) o un pronombre personal (12)), que funge como poseedor, al elemento nominal que equivale al poseído.

(11) nugwä nguye tain

pájaro ala roja

'Las alas del pájaro son rojas' 
(12) $t i$ gwe ti grü tu-ri

1SG ERG 1sg tío ver-IRREMOTOSPECTIV()

'Yo vi a mi tío'

La posesión también se expresa mediantc cl marciador gwe pospuesto al poseedor. En este caso, el orden se invicrle y se transforma en poseído-poseedor. Véanse (13) y (14):
(13) kö nun gwe otochí
cuerda nosotros POS corta
'Nuestra cuerda es corta'
(14) dän ere nun gwe ropa mucha nosotros POS 'Tenemos mucha ropa'

Aun cuando el orden de la lengua sea SOV y el poseedor precede al sustantivo, el adjetivo lo sigue; es decir, que es posnominal cuando lo esperable es que lo preceda. Al parecer, la lengua guaymí no es una lengua rectum-regens estricta.

También es posible que los demostrativos modifiquen pronombres personales como en el caso de (15), cosa que también ocurre en bocotá (Quesada, comunicación personal).

\section{(15) kwe se roro nga \\ 3sG DEM cuello largo \\ 'Él tiene el cuello largo'}

El orden del numeral es posnominal, y también manifiesta un orden alternativo, donde el clasificador aparece en posición posverbal, o sea que en guaymí existe el fenómeno de la frase nominal escindida (16). Sin embargo, las oraciones con el orden sustantivo-adjetivonumeral (preverbal) son consideradas extrañas y las que tienen el 86 
adjetivo después del verbo se rechazan por agramaticales; la escisión del sintagma nominal solo permite la extrapolación del numeral.

(16) Kwe kwi judä kwit-i kwa-ti

3s gallina huevo comer-IRREMOTOSPECTIVO CFL.REDONDO-uno

'Él se comió un huevo de gallina'

El guaymí manifiesta un sistema de clasificación de los objetos contados según una serie de características culturalmente concebidas.

Los numerales básicos son los siguientes:

$\begin{array}{lllc}\mathrm{ti}= & 1 & \mathrm{tí}= & 6 \\ \mathrm{bu}= & 2 & \text { kügü }= & 7 \\ \mathrm{mä}= & 3 & \mathrm{kwä}= & 8 \\ \text { bägä }= & 4 & \text { ögän }= & 9 \\ \text { rigué }= & 5 & \text { jätä }= & 10\end{array}$

Los clasificadores numerales son prefijos de los numerales básicos. Designan, por ejemplo, objetos redondos como una gallina ( $k w a-t i)$, animales u objetos largos como una serpiente ( $k r a-t i)$ y paquetes o grupos de personas (ketéi-ti). El resto de los clasificadores son: hojas (ka-ti), personas (i-ti), árboles y plantas (da-ti), cuartas (tai$t i)$, veces (ba-ti), racimos (kidei-ti), filas (jirei-ti), ropa (ötöi-ti), objetos delgados y sonoros, como cuchillos y astillas, (kun-di), dinero panameño (maná-ni), atados grandes (dui-ti), días (köböi-ti), días a partir de mañana (jetébe), días desde ayer (jädrin) y la distancia de la punta del dedo con el brazo extendido hasta el hombro contrario (ngwrai-ti). Todos los ejemplos anteriores indican uno.

\section{La frase verbal}

Aparte del verbo, dentro de la frase verbal pueden existir objetos directos (para las oraciones transitivas), adverbios (17), sintagmas posposicionales (18), la partícula de reflexividad (19 y 20) y el 
morfema libre de negación (21). Como el orden básico de la lengua es $\mathrm{SOV}$, los objetos directos son preverbales, el morfema de reflexividad antecede al objeto directo (en el caso de la posesión externa, como en (20)), mientras que los sintagmas posposicionales y adverbios son típicamente posverbales.

(17) mä ngobó nguetregä krübate

2sG hijo llorar.PRS mucho

'Tus hijos lloran mucho'

(18) mä jata-í jetebe María ben

2sG venir-FUT mañana María con

'Usted vendrá mañana con María'

(19) kwe tä ja bätete $\tilde{n} \ddot{o}$ ogwä kri te 3SG EXIST REFL lavar.PRS agua pozo grande en 'Ella se está lavando en la laguna grande'

(20) kwe tä ja mrögara kegue

3SG EXIST REFL cadera rascar.PRS

'Él se está rascando la cadera'

(21) kwe ie ti meye kä ñaka gain 3SG DAT 1SG mamá nombre NEG saber.PRS 'Él no se sabe el nombre de mi mamá'

El morfema de negación posee tres alomorfos, los cuales son $\tilde{n} a k a, \tilde{n} u k w a$ y $\tilde{n} a$. El alomorfo $\tilde{n} a$ tiende a situarse antes del marcador gwe (22), en oraciones transitivas, aunque es posible encontrarlo también precediendo al verbo en oraciones intransitivas, como en (23). También se observa el alomorfo ñukwa precediendo verbos en oraciones con valor impersonal, como en (24). En (21) el alomorfo ñaka se encuentra entre el objeto directo y el verbo, en una oración con un 88 
sujeto dativo. La distribución de estos alófonos requiere más investigación.

(22) Meri ña gwe $i \quad u-r i$

Mujer NEG ERG maíz moler-IRREMOTOSPECTIVO

'La mujer no ha molido maíz'

(23) nugwä niguengä, gwá ña gain

pájaro volar.PRS pez NEG saber.PRS

'Los pájaros vuelan, los peces no saben'

(24) tsö ñukwa täen

luna NEG ver.PRS

'La luna no se ve'

\section{Relaciones gramaticales básicas}

En una oración transitiva prototípica guaymí, el sujeto se puede reconocer como aquel sintagma nominal que codifica al agente y que se encuentra en posición inicial de oración. Sin embargo, se pueden mencionar otras dos propiedades que lo caracterizan: es el referente del morfema de reflexivización, como se aprecia en (19 y 20) y es el destinatario de la imperativa que se elide, en términos de Dixon, como en (9) y $(26)^{29}$. Existen, además, los sujetos dativos, los cuales aparecen en oraciones que no son prototípicamente transitivas, con sujetos no agentes, los cuales reciben una marca similar a la que reciben los benefactivos y experientivos; véanse (21) y (25).

(25) kwe ie ngitrá kun-i

3SG DAT cuchillo encontrar-IRREMOTOSPECTIVO

'Él encontró el cuchillo'

$\overline{29}$ R. M. W. Dixon, Ergativity (Cambridge, Cambridge University Press, 1994) 131. 
En cuanto a los objetos directos, Éstos condilicinl piacicutes y preceden al verbo. Esta posición tiende a ser cilsi cxullusivil; c.jemplo

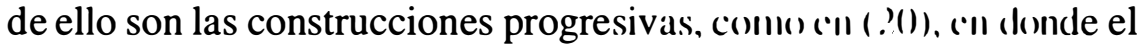
complejo verbal se ve separado por el compleminlolo directo para mantener la configuración OV.

Finalmente, la categoría de dativo está expresirlla c'll unl adjunto que puede codificar roles semánticos de bencl ic livo ( III N) o experientivo (EXP) y que se encuentra marcado por el morlimil ic', el cual se puede reducir fonéticamente a $e$. La posición cinnónicia del dativo es posverbal.

(26) kwi judä biaín kwä-jätä $\quad$ hi kil-bu gallina huevo dar.PRS CFL.REDONDO-dic\%, más ( II..RI:IOONDO-dos $t i$ ie 1SG DAT

‘Déme esos doce huevos de gallina!'

\section{Conclusión}

Se han presentado, de una manera global, las características gramaticales y tipológicas generales de la lengua guaymí. Además, se ha mostrado el estado de la lengua en cuanto a su vitalidad en Costa Rica y Panamá. El interés ha sido panorámico más que exhaustivo, por tratarse de una etapa de la larga tarea de descripción de la estructura gramatical de esta lengua. Entre otras cosas, queda pendiente un estudio más pormenorizado sobre el marcador gwe que pueda definir con certidumbre acerca de la pertenencia de la lengua guaymí a un sistema ergativo-absolutivo, a un sistema de ergativo extendido u otro. Asimismo, el sistema temporo-aspectual y fenómenos de estructura de la información son parte de nuevas tareas por llevar a cabo. 